\title{
Re: Comparison of Microdissection Testicular Sperm Extraction, Conventional Testicular Sperm Extraction, and Testicular Sperm Aspiration for Nonobstructive Azoospermia: A Systematic Review and Meta-Analysis
}

\author{
Bernie AM1, Mata DA2, Ramasamy R3, Schlegel PN4 \\ 1Weill Cornell Medical College, Department of Urology, New York, USA \\ 2 Harvard Medical School, Brigham and Women's Hospital, Department of Pathology, Massachusetts, USA \\ 3University of Miami Faculty of Medicine, Department of Urology, Miami, Florida \\ 4Weill Cornell Medical College, Department of Urology, New York, USA
}

Fertil Steril. 2015;104:1099-1103.e1-3. doi: 10.1016/j.fertnstert.2015.07.1136. Epub 2015 Aug 8.

\section{EDITORIAL COMMENT}

In contemporary medicine, testicular sperm extraction (TESE) and using testicular sperm for intracytoplasmic injection is the only fertility treatment in men with nonobstructive azoospermia (NOA). Different sperm retrieval techniques have been used to find a single sperm from the testes of men with NOA. In this study, three sperm retrieval techniques, microdissection TESE (micro TESE), conventional TESE (cTESE), and testicular sperm aspiration (TESA) were compared for yielding successful sperm recovery. Fifteen studies with total of 1890 patients were eligible for inclusion of meta-analysis. These studies were published between 1997 and 2012 and took places - six in Asia, four in Europe, three in North America and two in Africa. Using different tissue processing techniques and the patient heterogeneity that exists in the population of men diagnosed with NOA are the limitations of this study. In conclusion, meta-analysis of the studies has shown that performance of micro-TESE was higher compared with cTESE and performance of cTESE was higher compared with TESA for successful sperm retrieval.

Emre Bakırcıoğlu MD

\section{Andrology}

doi: $10.4274 /$ jus.2016.01.006

\section{Re: The Who, How and What of Real-World Penile Implantation in 2015: The PROPPER Registry Baseline Data}

Henry GD1, Karpman E2, Brant W3, Christine B4, Kansas BT5, Khera M6, Jones L7, Kohler T8, Bennett N9, Rhee E10, Eisenhart E11, Bella AJ12

1Regional Urology, Shreveport, Louisiana, USA

2El Camino Urology Medical Group, Mountain View, California, USA

3University of Utah Faculty of Medicine, Department of Urology, Salt Lake City, Utah, USA

4 Urology Centers of Alabama, Birmingham, Alabama, USA

5The Urology Team, Austin, Texas, USA

6Baylor College of Medicine, Houston, Texas, USA

7 San Antonio Urology, San Antonio, Texas, USA

8Southern Illinois University Faculty of Medicine, Springfield, Illinois, USA

9Lahey Hospital and Medical Center, Burlington, Massachusetts, USA

10Kaiser Permanente, San Diego, California, USA

${ }^{11 R e g i o n a l ~ U r o l o g y, ~ S h r e v e p o r t, ~ L o u i s i a n a ~}$

12University of Ottawa Faculty of Medicine, Department of Urology and Surgery, Ontario, Canada

J Urol. 2016;195:427-433. doi: 10.1016/j.juro.2015.07.109. Epub 2015 Aug 17. 